\title{
Chapter 6 \\ Improvement of Wind Turbine Blade Performance by Means of Rod Vortex Generators
}

\author{
Javier Martinez, Pawel Flaszynski, Piotr Doerffer, and Oskar Szulc
}

\begin{abstract}
Wind turbines are complex energy conversion fluid-flow machines which entail coupled aero-mechanical issues. From an aero-acoustical point of view, wind turbine blades present two main problems: first, a reduced aerodynamic performance due to flow separation, and second, the level of noise emissions. Flow separation appears on the blade as a result of high angles of attack causing a decrease in the aerodynamic efficiency. In this chapter, the application of rod vortex generators (RVGs) to control and decrease the flow separation-by the creation of streamwise vorticity on the blade - is presented. The NREL Phase VI wind turbine rotor and the S809 airfoil are used as reference cases. The validation of NREL Phase VI model rotor against experimental data is found to be satisfactory. A study into the effects of RVGs' chordwise location and spanwise distance is presented for selected cases and a range of inflow conditions. It is shown that the proposed RVGs lead to an improvement of the aerodynamic performance, and can be successfully applied by the wind energy industry.
\end{abstract}

\subsection{Introduction}

The wind energy sector has experienced rapid growth in the last few decades. The developments in the materials science, engineering and allied fields have ushered in turbines of increasing sizes; turbines with rotor diameters of up to $160 \mathrm{~m}$ (Vestas V164 $8 \mathrm{MW}$ ) have been developed, and the current trend of increasing sizes is expected to continue in the future (Bak et al. 2012).

The increasing sizes of wind turbines pose new challenges for engineers. One particular challenge is that large rotor dimensions result in non-uniform inflow conditions along the blade span-which leads to increased flow separation even after the application of traditional flow control approaches. As a consequence of

\footnotetext{
J. Martinez $(\square) \bullet$ P. Flaszynski • P. Doerffer • O. Szulc

Aerodynamics Department, Institute of Fluid Flow Machinery, Polish Academy of Sciences, ul. Fiszera 14, 80-231 Gdańsk, Poland

e-mail: javier.martinez@imp.gda.pl; pawel.flaszynski@imp.gda.pl; piotr.doerffer@imp.gda.pl; oskar.szulc@imp.gda.pl
} 
the boundary layer separation, the air flow on the blade is detached leading to increased aerodynamic losses and noise generation (Wagner et al. 2012). In order to reduce the flow separation, different flow control devices have been developed and implemented on the blades during the last few decades (Van Dam et al. 2008). Several flow control devices, such as trailing edge flaps, microtabs, microflaps, plasma actuators, active flexible walls or vortex generators, have been proposed in literature (Van Dam et al. 2007, 2008; Andersen et al. 2010; Johnson et al. 2010; Nelson et al. 2008; Gao et al. 2015). Of these, the current chapter focuses on vortex generators.

Vortex generators (VGs) are located in the boundary layer in order to create a streamwise vorticity, which influences the transport of momentum in the direction normal to the wall. The fluid with higher momentum transported to the near wall zone energizes the boundary layer. This leads to increased shear stresses and higher resistance to the adverse pressure gradient effect which results in reduction or even complete removal of the separation.

The first VG investigations were conducted by Taylor (1947) in the late 1940s. These initial devices, of height $(h)$ higher or equal to the boundary layer $(\delta)$, were called vane vortex generators (VVGs). During the 1950s, air jet vortex generators (AJVGs) were developed. It was found that they could be considered as an effective alternative to traditional VGs for separation reduction (Wallis and Stuart 1962). In the 1980s, Taylor's VGs were replaced by smaller devices, with the introduction of low profile VGs $(h / \delta<0.65)$ (Rao and Kariya 1988); these devices generate lower disturbance and flow non-uniformity outside boundary layer in comparison with the classical vanes as they are submerged inside the boundary layer. As an effect, the drag is lower and the effectiveness of the flow control method can be higher. However, these devices generally need to be located closer to the critical zone than classical vanes due to faster decaying streamwise vorticity (Lin 2002).

In the last few decades VGs have been widely researched and new concepts of non-solid VGs - such as synthetic jets or pulsed jets (Lin 2002; Wiltse and Glezer 1993; Amitay et al. 1998; McManus et al. 1994; Kostas et al. 2007)—have been proposed. It has been demonstrated that active VGs provide similar benefits as passive VGs, but with a major added advantage: when they are not functional, there is no increment in the parasitic drag (Paul et al. 2013). Despite the clear benefits of active VGs, different configurations of vane-type VGs are still a commonly implemented solution on wind turbine blades.

This chapter focuses on the investigation and application of a new type of VG, called Rod Vortex Generator (RVG) (Doerffer et al. 2009), on wind turbine blades. Optimized RVGs have demonstrated a capability to generate similar streamwise vortex intensity as the one generated by the AJVGs (Tejero et al. 2015). Furthermore, RVGs do not require complex installation, as is the case with AJVGs. In contrast to the traditional vane-type VGs, RVGs can be combined with Micro-ElectroMechanical Systems (MEMS) technology, thus allowing for activation during the system operation if the conditions require its application.

RVGs can be defined by five parameters: diameter (D), height (h), skew angle $(\theta)$, pitch angle $(\alpha)$ and spacing $(W)$, as shown in Fig. 6.1. The first four parameters 


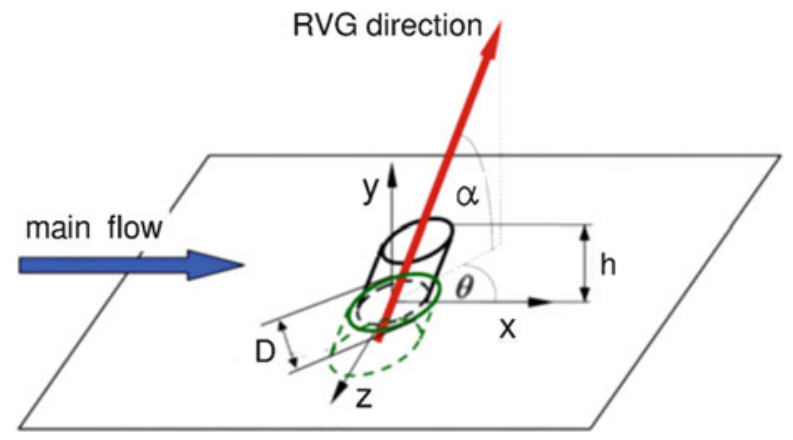

Fig. 6.1 Rod vortex generator configuration

define the geometry and orientation of the rod, while the spacing (W) refers to the spanwise distance between rods. The orientation angles $(\theta$ and $\alpha)$ are optimized to obtain the maximum streamwise vorticity. The rod diameter and the height are proportional to the boundary layer thickness. The chordwise location of the RVG along the blade $\left(x_{R V G} / c\right)$ is also a crucial parameter which has to be taken into account.

The application of RVGs on wind turbine blades, as a flow control device to improve aerodynamic performance, is analysed for the NREL Phase VI wind turbine rotor. The NREL turbine was selected because of the large amount of experimental data available (Hand et al. 2001) and the detailed geometry description (Lindenburg 2003) which is highly advantageous for the validation step.

\subsection{Flow Solver}

The numerical investigations have been carried out by means of FINETM/Turbo Numeca. The compressible, mass-weighted RANS equations are solved adopting a preconditioning scheme (Hakimi 1997; Choi and Merkle 1993). A full multigrid technique is applied in order to increase the convergence rate. Spatial discretization is based on a cell centred control volume approach, and a 2nd order central difference scheme with scalar artificial dissipation formulated by Jameson (1991) is applied. The results presented in this chapter are obtained for turbulence models (Spalart and Allmaras 1992), k- $\omega$ SST (Menter 1994) and Explicit Algebraic Reynolds Stress Model (EARSM) (Menter et al. 2012). A perfect gas model is employed for closure of the system of differential equations. The dynamic viscosity is calculated using the Sutherland's law (Sutherland 1893). 


\subsection{The NREL Phase VI Wind Turbine Rotor}

\subsubsection{Experimental Set-Up}

The computational model is validated by the comparison of numerical simulations results with the experimental data available in the National Renewable Energy Laboratory (NREL) database for the Unsteady Aerodynamics Experiment Phase VI (Hand et al. 2001). The geometry of the investigated configuration is shown in Fig. 6.2.

The two-bladed rotor was located in the $24.4 \mathrm{~m} \times 36.6 \mathrm{~m}$ NASA Ames Wind Tunnel and tested under several different inflow conditions, with velocities ranging from 0 to $25 \mathrm{~m} / \mathrm{s}$. The stall regulated rotor was equipped with two linearly tapered, non-linearly twisted blades of radius $\mathrm{R}=5.209 \mathrm{~m}$ with rounded tip caps (Lindenburg 2003). The tapered region of the blades starts at $r / R=0.25$ with a chord length $c=0.737 \mathrm{~m}$, and ends at the tip, $\mathrm{c}=0.357 \mathrm{~m}$. The non-linearly twisted blade, defined with respect to the $\mathrm{r} / \mathrm{R}=0.75$ section and considered positive towards feather, varies from $20.05^{\circ}$ at $\mathrm{r} / \mathrm{R}=0.25$ to $-1.82^{\circ}$ at tip.

During the sequence $S$ of the experimental campaign (Hand et al. 2001), the blade tip pitch angle was set to $3^{\circ}$ with respect to the rotor plane, the rotational speed to $72 \mathrm{rpm}$, and the cone angle to $0^{\circ}$. Static pressure measurements were obtained at five spanwise locations $(\mathrm{r} / \mathrm{R}=0.3,0.466,0.633,0.80$ and 0.95$)$. Yaw angles were varied from 0 to $180^{\circ}$, but only the results from the $0^{\circ}$ yaw angle case were harnessed for validation purposes. In the present work, only wind speeds of 5,7,10 and $13 \mathrm{~m} / \mathrm{s}$ are considered. Table 6.1 summarizes the operational conditions for the computed cases (Hand et al. 2001; Sørensen and Michelsen 2000).
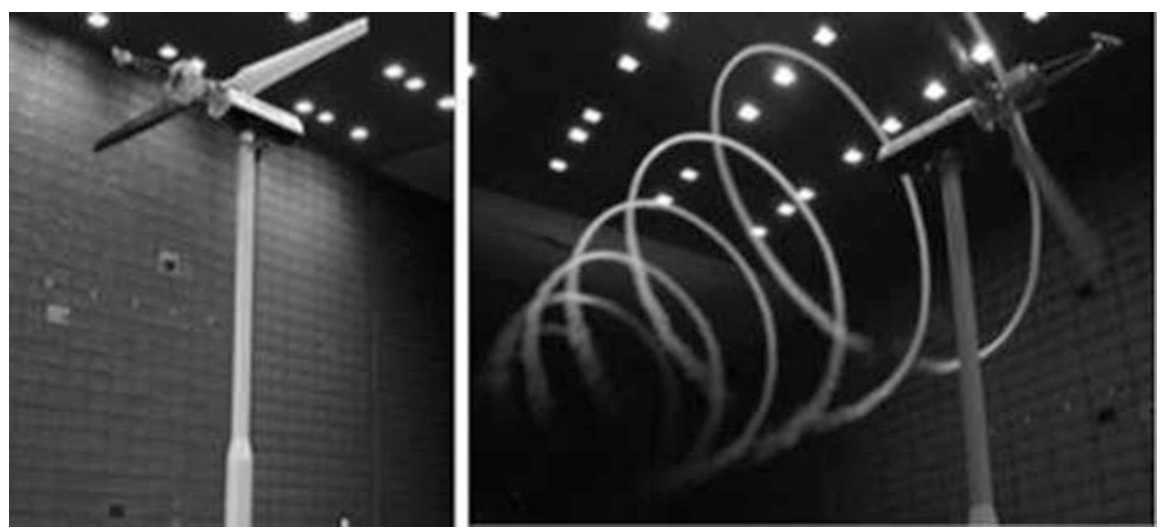

Fig. 6.2 NREL Phase VI wind turbine at NASA Ames (Lindenburg 2003) 
Table 6.1 NREL Phase VI operating conditions

\begin{tabular}{c|l|l|l}
\hline Wind speed $[\mathrm{m} / \mathrm{s}]$ & Density $(\rho)\left[\mathrm{kg} / \mathrm{m}^{3}\right]$ & RPM & Temperature $[\mathrm{K}]$ \\
\hline 5.0 & 1.243 & 71.7 & 284.25 \\
\hline 7.0 & 1.246 & 71.9 & 284.25 \\
\hline 10.0 & 1.246 & 72.1 & 284.15 \\
\hline 13.1 & 1.227 & 72.1 & 286.85 \\
\hline
\end{tabular}

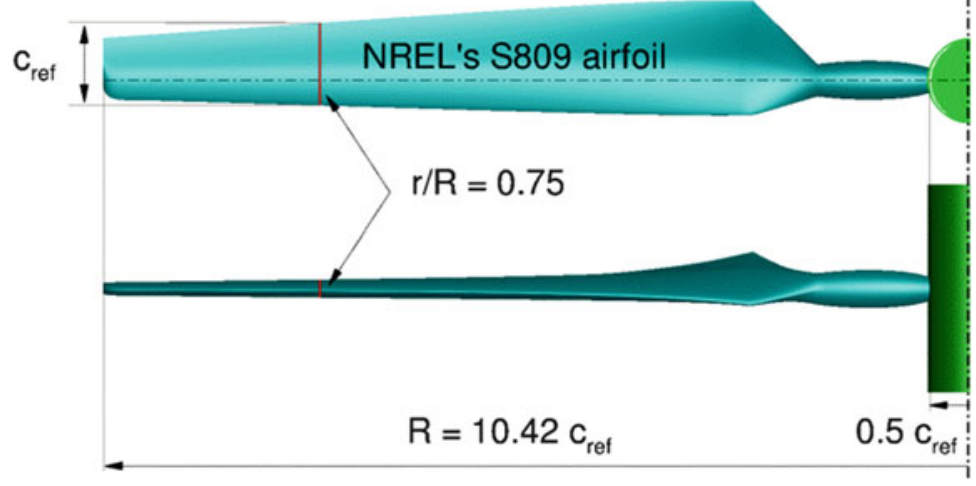

Fig. 6.3 NREL Phase VI rotor model

\subsubsection{Numerical Modelling}

A numerical model is created for the NREL Phase VI rotor. The section $r / R=0.75$ was taken as the reference section, and the local chord was set to unit $\left(c_{\text {ref }}=1\right)$. The blade radius to reference chord length ratio $(A R \approx 10.42)$ was kept constant in comparison with the full scale model. Thus the blade radius was rescaled to $R=\mathrm{AR} \cdot c_{r e f}$. The pitch and sectional twists were not affected by the process. The nacelle was modelled as a cylinder with radius $0.5 \cdot c_{\text {ref }}$ (Fig. 6.3) (Martinez et al. 2015a).

The rotational periodicity of the flow allows the use of a computational domain defined by a half of cylinder with a single blade in the middle. Farfield boundary conditions are applied at the outer cylinder, located at a distance of 3 blade radius $(R)$ from the rotor axis. The inlet and outlet are located at a distance equal to $3 \cdot R$ from the rotor plane. At the inlet, a uniform velocity distribution in normal direction to the rotor plane is applied, while at the outlet, atmospheric static pressure is imposed. The blade is defined as a non-slip wall, while at the cylindrical surface (hub) an Euler wall (no shear stress at the wall) condition is applied. This enables one to save resources and computational time (Fig. 6.4).

The computational domain consists of 8.8 million cells distributed amongst 76 hexahedral full-matching connection blocks. The structured grid is of $\mathrm{C}$ type in the crosswise direction, and $\mathrm{H}$ type in the spanwise and freestream directions, as shown 


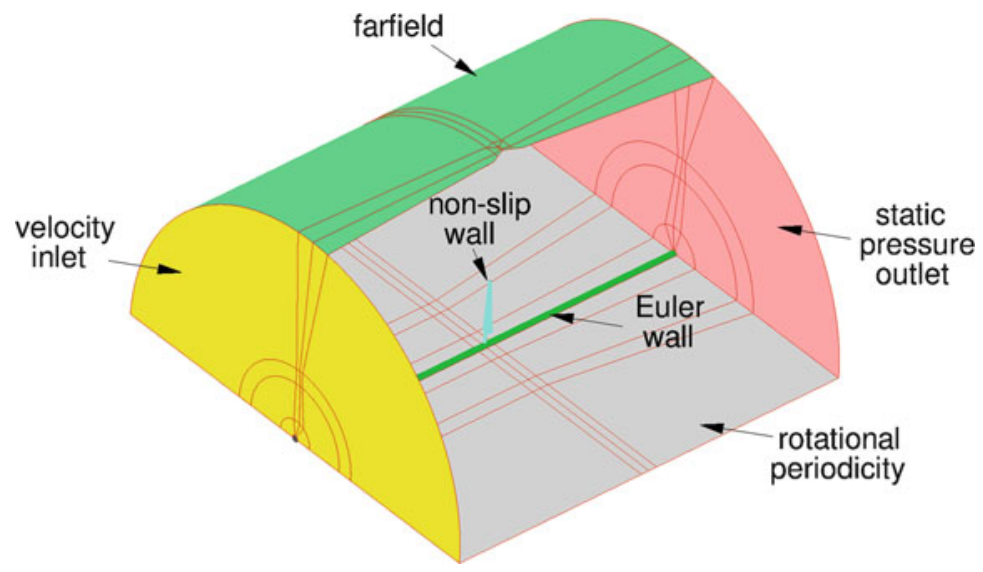

Fig. 6.4 NREL Phase VI rotor computational domain and boundary conditions
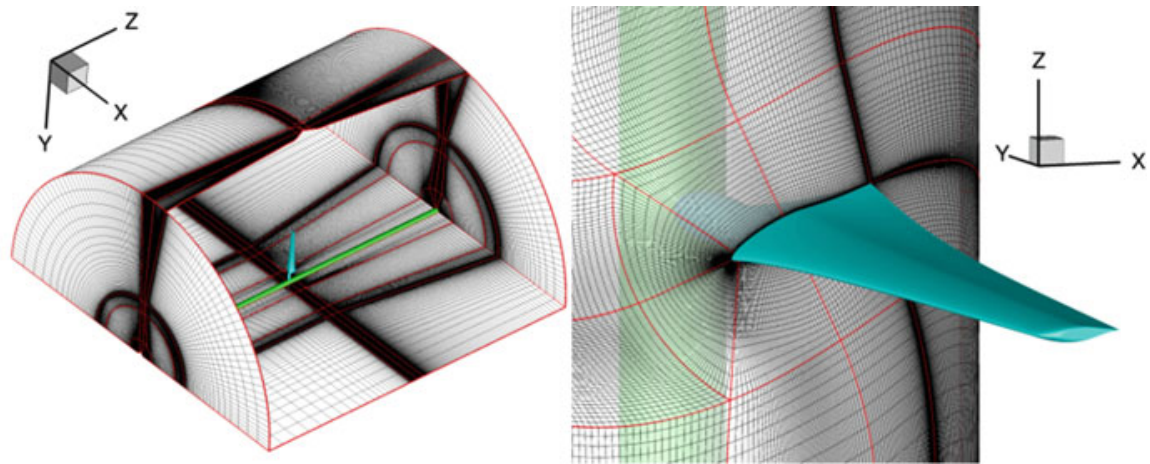

Fig. 6.5 NREL Phase VI rotor C-H-H grid

in Fig. 6.5. The blade surface is discretized by 294 nodes around the airfoil and 91 nodes in the spanwise direction. The non-dimensional distance of the first cell layer close to the wall is of the order of $y^{+} \sim 1$.

\subsubsection{Numerical Results}

The numerical simulations for the NREL wind turbine rotor show good agreement with measurements of the total thrust and torque. The only difference noted is for a wind speed $10 \mathrm{~m} / \mathrm{s}$ (Fig. 6.6), where torque is over-predicted (Martinez et al. 2015b).

It is shown in Fig. 6.6 that the predictions obtained by means of 2-equation $\mathrm{k}-\omega$ SST and EARSM models are almost identical. One has to emphasize that in case of 

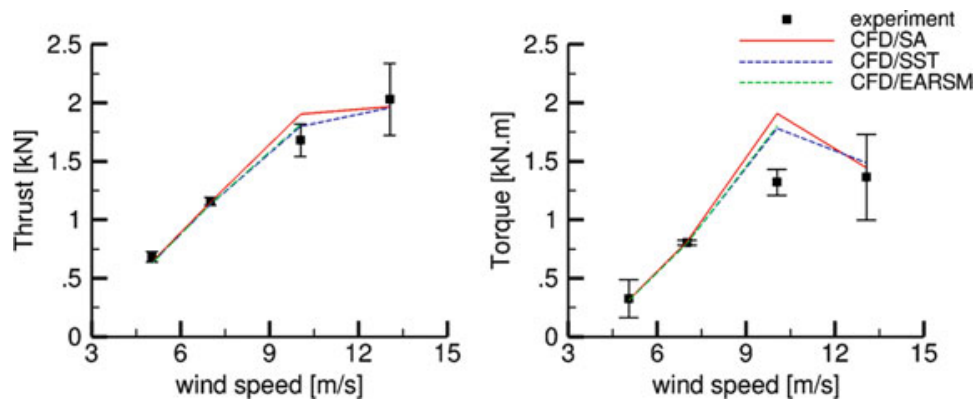

Fig. 6.6 Integrated thrust (left) and torque (right) for NREL Phase VI rotor

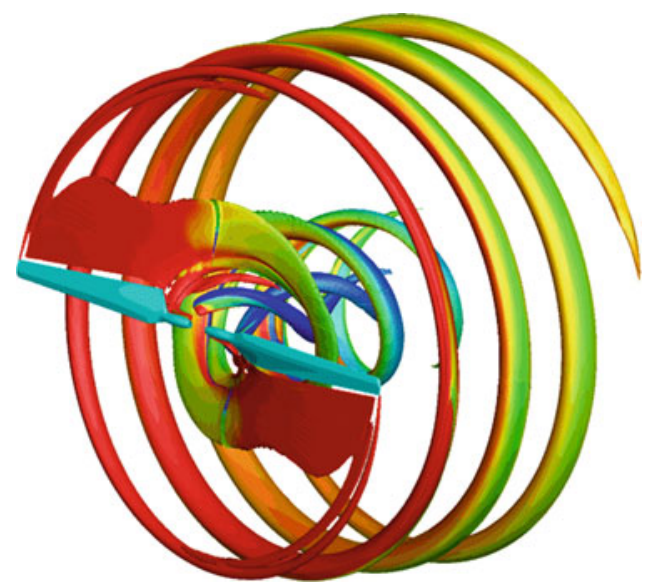

Fig. 6.7 NREL Phase VI rotor aerodynamic wake at $5 \mathrm{~m} / \mathrm{s}$

thrust measurements, computed values are within the measurement accuracy. The discrepancy is higher for the SA model.

A Q-criterion (coloured by the vorticity magnitude) visualization of the rotor wake (Fig. 6.7) was extracted from the numerical solution for an inflow wind velocity of $5 \mathrm{~m} / \mathrm{s}$, and the one equation Spalart-Allmaras turbulence model. The rotor wake created by wind turbines is closely related to the amount of energy extracted from the wind, and its prediction is of primary importance in the assessment of performance and power. An example of the wake prediction by numerical model, described in more details in Martinez et al. (2015a, b), is shown in Fig. 6.7.

For validation purposes a more detailed analysis of the flow properties (particularly, flow attachment state and local blade loading) is needed. The blade surface streamlines and friction coefficient $c_{f}$ provide information of utmost importance regarding the flow attachment state and developed flow patterns. The blade loading analysis is given by the sectional chordwise pressure coefficient $c_{p}$, and spanwise 
normal $c_{N}$ and tangential $c_{T}$ force coefficients distributions. The friction coefficient $c_{f}$ is defined as:

$$
c_{\mathrm{f}}=\frac{\mu \frac{\mathrm{du}_{\mathrm{s}}}{\mathrm{dn}}}{0.5 \rho \mathrm{U}_{\infty}^{2}}
$$

where $\mathrm{u}_{\mathrm{s}}$ is the component of cross sectional velocity tangential to the surface, $\mathrm{n}$ is the direction normal to the blade surface, $U_{\infty}$ the cross sectional reference velocity, $\mu$ the freestream viscosity and $\rho$ the density.

The friction coefficient $c_{f}$ along with velocity streamlines are shown in Fig. 6.8 for the 4 wind speeds ranging from 5 to $13 \mathrm{~m} / \mathrm{s}$. Contours for $c_{f}$, obtained only from the Spalart-Allmaras turbulence model, are shown. Analogous behavior is observed in case of the two equation turbulence models.

It is observed that in the case of 5 and $7 \mathrm{~m} / \mathrm{s}$ wind speeds, the flow stays fully attached-except for the inner section, where the cylindrical shape (close to root) induces local separation. At $10 \mathrm{~m} / \mathrm{s}$, according to the SA model prediction, the previous local separation moves towards the outer span of blade until $r / R=0.95$. The plot for the wind speed of $13 \mathrm{~m} / \mathrm{s}$ reveals that the flow is completely detached on the suction side, except for a very narrow region at the blade tip. Further increasing of the wind speed causes the detached flow area to increase.

An example of pressure coefficient distributions $c_{p}$ located at four blade cross sections $(\mathrm{r} / \mathrm{R}=0.30,0.466,0.80$ and 0.95$)$ and the non-dimensional force coefficient $c_{N}$ are compared to the experimental data in the Figs. 6.8 and 6.9 for wind

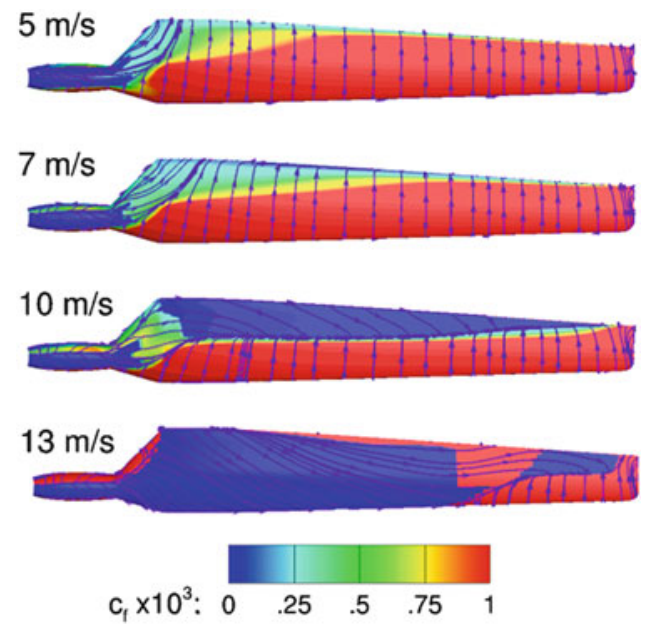

Fig. 6.8 Skin friction coefficient $c_{f}$ and surface streamlines (suction side) 

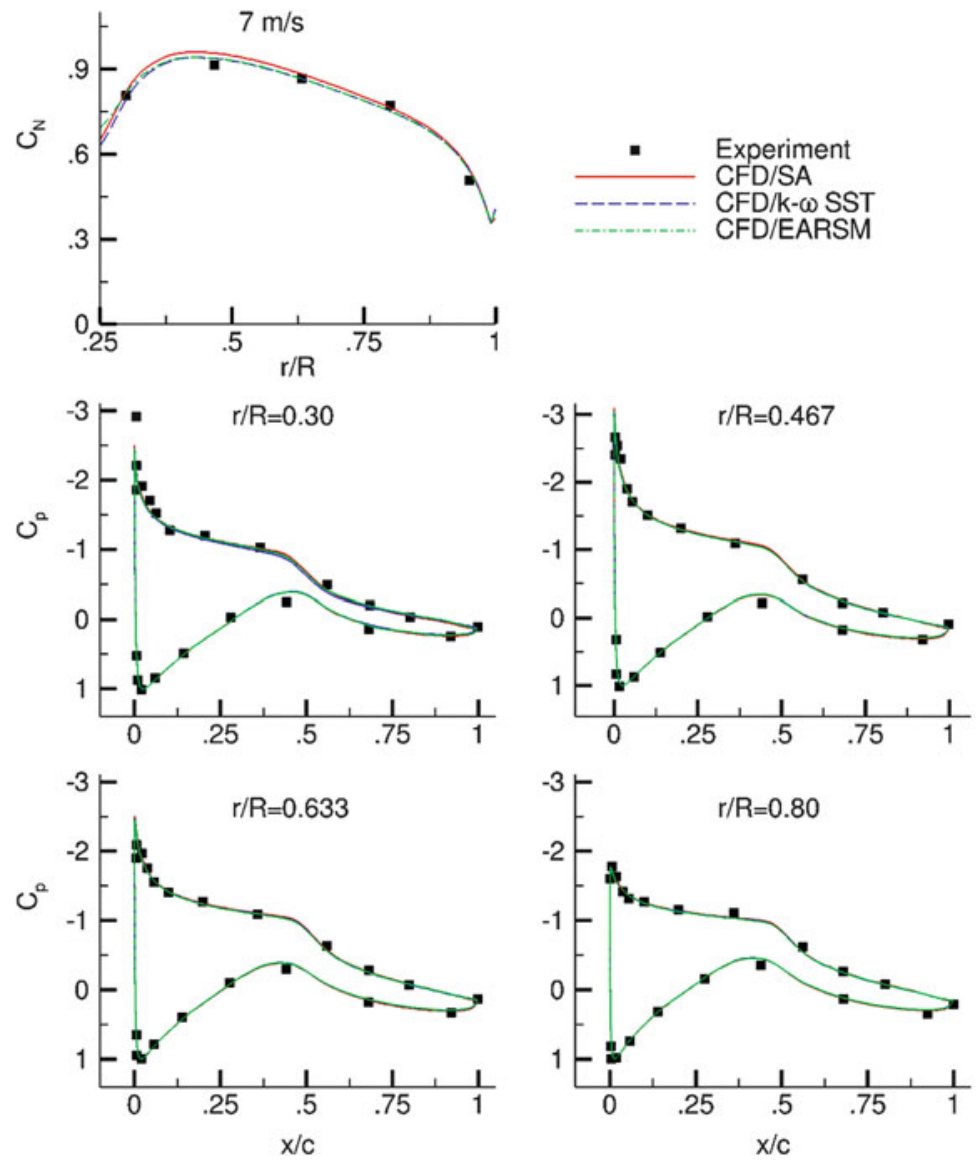

Fig. 6.9 Pressure coefficient $\boldsymbol{c}_{p}$ and normal force $\boldsymbol{c}_{N}$ distributions at $7 \mathrm{~m} / \mathrm{s}$

speeds of 7 and $10 \mathrm{~m} / \mathrm{s}$, respectively. The sectional normal force coefficient $c_{N}$ distributions predictions were obtained by the integration of the $c_{p}$ distributions along each one of the 100 cross sections employed. Figure 6.9 shows an exemplary agreement with the experimental data. A slight difference among models is observed at the $30 \%$ cross section.

As the wind speed increases to $10 \mathrm{~m} / \mathrm{s}$ the prediction of SA model fails to reproduce the normal force coefficient distribution, although the k- $\omega$ SST and EARSM models predictions are still close to the experimental data (Fig. 6.10). The $10 \mathrm{~m} / \mathrm{s}$ case shows important discrepancies between the $c_{p}$ distribution predicted and the experimental data for the $46.6 \%$ span section. The experimental data seems to show a local separation at this section, which is not predicted by numerical simulations, while a reasonably good agreement with the experimental data is obtained in the remaining sections. 

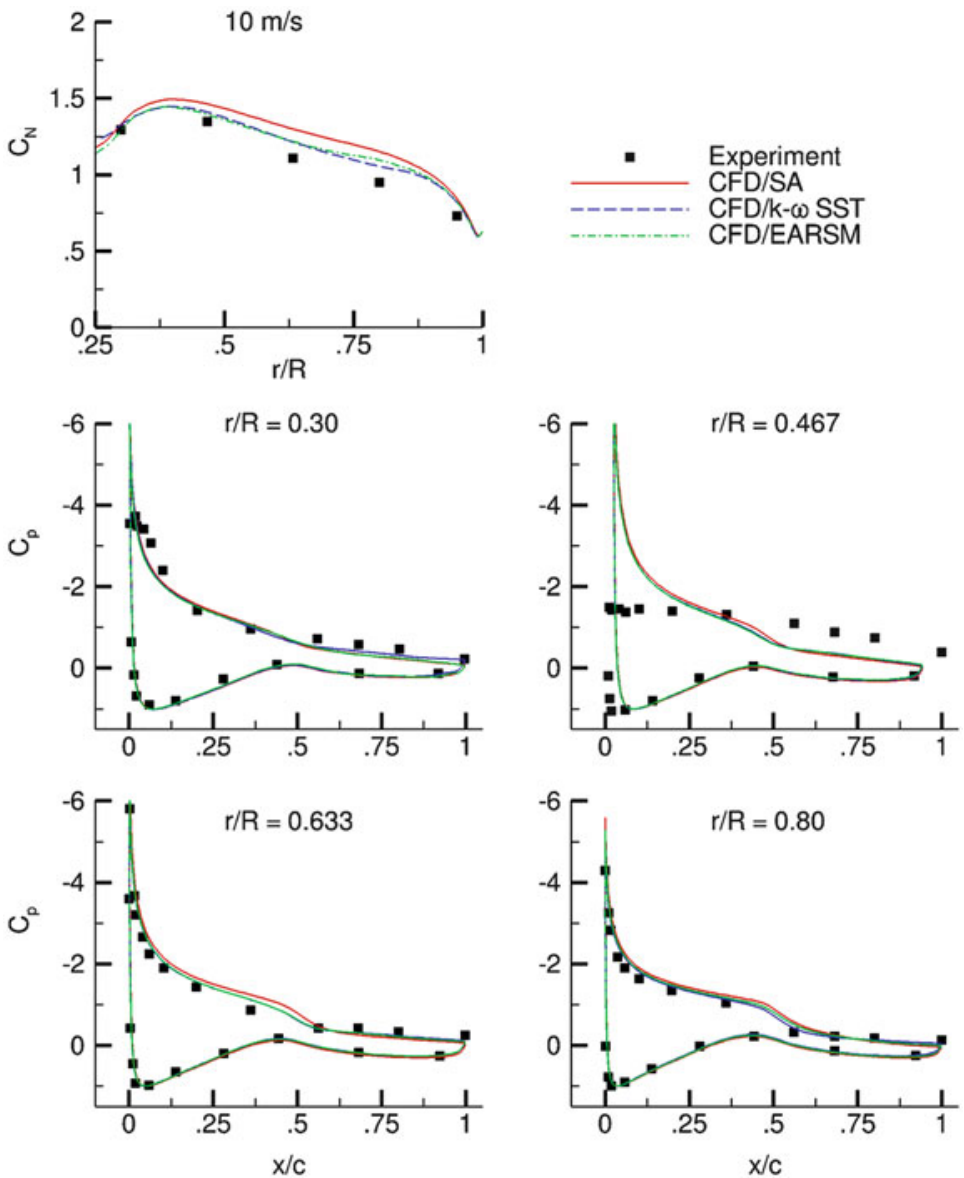

Fig. 6.10 Pressure coefficient $c_{p}$ and normal force distributions $c_{N}$ at $10 \mathrm{~m} / \mathrm{s}$

Disregarding the $46.6 \%$ span section, a reasonably good agreement between experimental and numerically predicted $c_{p}$ distributions has been shown for the turbine operating at wind speeds ranging from 5 to $13 \mathrm{~m} / \mathrm{s}$ (see Martinez et al. 2015a). Consequently, a flow structure similar to the displayed in Fig. 6.8 is expected to have been developed during the experimental campaign.

The optimum tip speed ratio is close to 5.4 (wind speed $7 \mathrm{~m} / \mathrm{s}$ ) (Martinez et al. $2015 \mathrm{a}, \mathrm{b}$ ); thus, as the wind speed increases beyond the optimum, the flow separation comes out on the blade, affecting the rotor performance. It influences the rising flow structure complexity, and causes difficulties with the prediction accuracy of the solver. The area of detached flow in the $10 \mathrm{~m} / \mathrm{s}$ case is quite susceptible of being decreased by means of rod vortex generator, thus improving the aerodynamic 
performance and delaying the separation as the wind speed increases. When the flow on the blade is totally detached, however, the application of RVGs is not expected to bring significant (if any) aerodynamic improvement. Besides, modern horizontal axis wind turbine rotors equipped with individual pitch control are more likely to operate with partially flow-separated blades, than in a strong stall situation. Thus the $10 \mathrm{~m} / \mathrm{s}$ case is chosen as a reference for a flow control study in wind turbines.

\subsection{The S809 Aerofoil (Clean Case)}

The NREL Phase VI rotor blades employ the $21 \%$ thickness S809 aerofoil. For this reason a validation study is performed for this aerofoil. Designed for wind turbines applications using the Eppler code (Eppler and Somers 1980), a short ramp of adverse pressure is present on the upper surface of this profile, downstream of the mid-chord. It promotes an efficient laminar to turbulent transition, followed by a low drag concave pressure recovery region (Somers 1997).

The S809 aerofoil was tested in the Ohio State University (OSU) and Delft University of Technology (DUT) facilities and experimental data is available for several angles of attack (Somers 1997) and Reynolds numbers (Ramsay et al. 1995). The validation against experimental data was performed for a Reynolds number of $1 \cdot 10^{6}$ to ensure that the analysis was representative of the studied flow around the NREL Phase VI blade.

The aerofoil surface was modelled as a non-slip wall. A C type grid was used, and the farfield was located at 40 chords from the aerofoil. The total computational cells amount to 0.3 million, distributed into 13 blocks. The $\mathrm{S} 809$ aerofoil surface is defined by $737 \times 145$ nodes in the wrap-around and normal directions, respectively (Fig. 6.11).
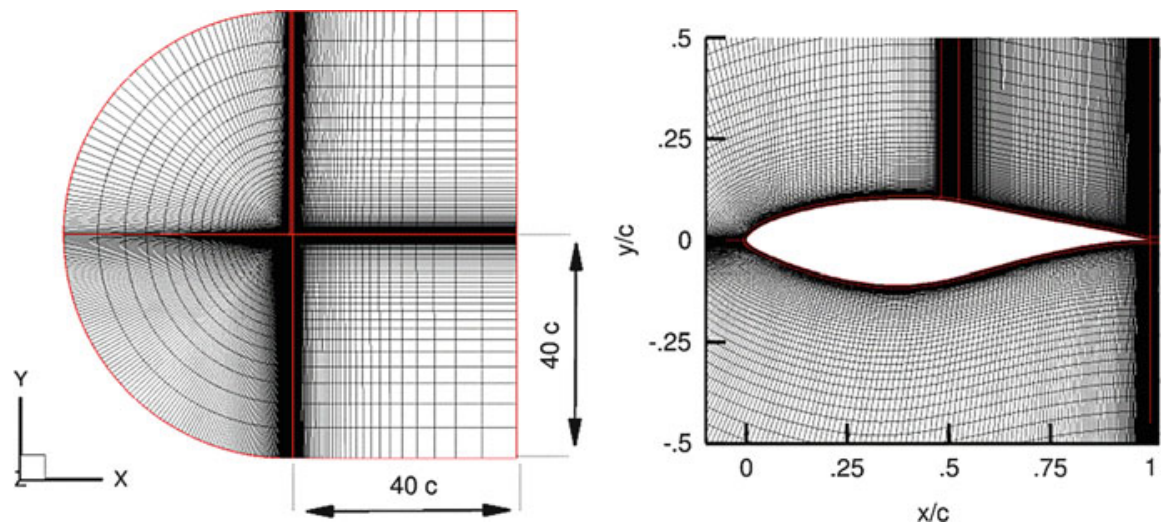

Fig. 6.11 C type grid around the S809 aerofoil 
Numerical RANS simulations were performed with and without a transition model. The $\gamma-\operatorname{Re}_{\theta}$ transition model is used. This model is based on two transport equations, one for intermittency, $\gamma$, and the second one for the transition momentum thickness Reynolds number, $\operatorname{Re}_{\theta}$. For simulations performed without the transition model, the flow is assumed to be fully turbulent and the one equation SpalartAllmaras and the two equation k- $\omega$ SST and EARSM models were employed. The gamma Reynolds theta transitional model $\left(\gamma-R_{\theta}\right)$ is adopted together with the k- $\omega$ SST and the EARSM turbulent models. Additionally, a Hakimi preconditioning scheme is applied for all the simulations.

Figure 6.12 depicts the evolution of lift $c_{l}$ and $\operatorname{drag} c_{d}$ coefficients with respect the angle of attack. It is apparent that numerical models have a good ability to predict the lift for moderate angles of attack, but overestimate the value for larger angles. Considering the drag prediction, the turbulence models including transition effect have proven to have a better prediction capability.

Comparisons between the predicted pressure coefficient $c_{p}$ distribution along the aerofoil and experimental data are shown in Fig. 6.13. Two selected angles of attacks, 4.1 and $13.3^{\circ}$, are compared. A pressure overestimation is given on the suction side by the SA model for the $13.3^{\circ}$ angle of attack, while the other two fully turbulent models provide a good agreement. For the $4.1^{\circ}$ angle of attack, both transition models are able to predict accurately the laminar separation bubble located just aft of the mid-chord on the suction side of the aerofoil.
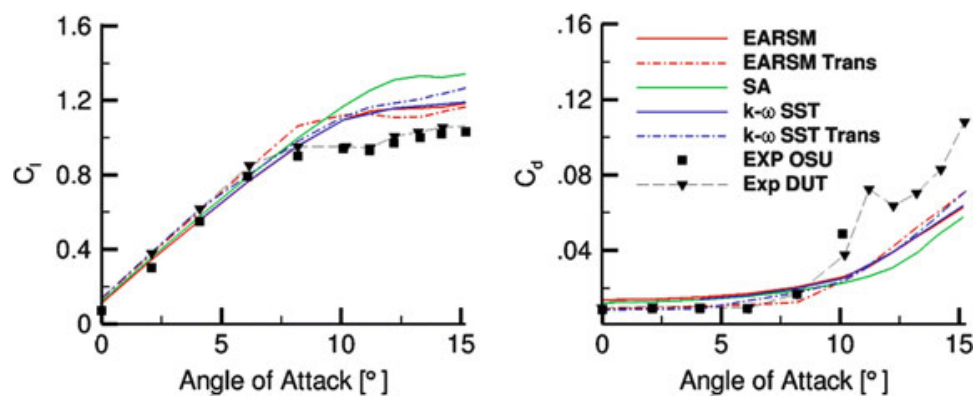

Fig. 6.12 Lift $c_{l}$ and drag $c_{d}$ coefficients versus angle of attack for S809 aerofoil
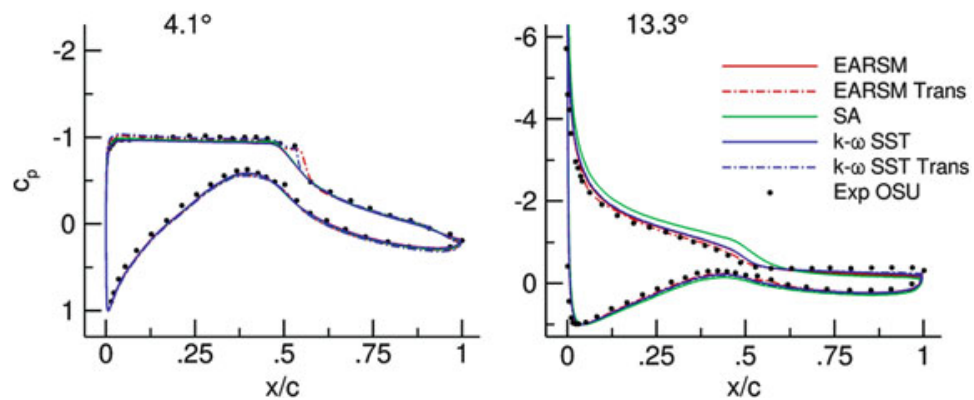

Fig. 6.13 Pressure coefficient $c_{p}$ distributions for S809 aerofoil 
The better prediction obtained with the 2 equation turbulent models compared to the 1 equation SA model, makes the former more adequate for the analysis of the complex flow structure developed inside the boundary layer when flow control devices are applied. Simulations for RVGs applied on blade are computationally demanding and only one turbulence model is selected for the further analysis.

The nonlinear constitutive relations of the EARSM model are capable of reproducing the anisotropy of the stress distribution-which is a very important feature beyond the possibilities of linear eddy viscosity turbulence models (SA, k- $\omega$ SST) — and makes this model even more suitable for the RVG implementation study.

\subsection{The S809 Aerofoil (Flow Control Case)}

\subsubsection{Basic Configuration}

Once the numerical methods have been favourably validated against experimental data for the NREL Phase VI wind turbine rotor and the S809 profile, the obtained results can be employed to the rod vortex generators design and optimization. In particular, the numerical predictions for the S809 aerofoil given by the fully turbulent EARSM model are employed for the dimensioning of the RVGs (h, D) and for the optimization of their chordwise location $\left(x_{R V G} / c\right)$ and spanwise distribution (W). Table 6.2 summarizes the parameters of the basic RVG configuration.

Four flow cases are taken into consideration for flow control investigations. All of them are selected for high inflow angles: 11.2, 12.2, 13.3 and $13.9^{\circ}$, with the separation onset located at $\mathrm{x} / \mathrm{c}=0.88,0.65,0.55$ and 0.53 , respectively. As the angle of attack increases the separation moves upstream to the mid-chord in the case of the highest loading considered.

The case of the $13.9^{\circ}$ inflow angle, where the most severe flow conditions exist, is taken as the reference and the rods are initially located slightly upstream of the separation onset $\left(x_{R V G} / c=0.50\right)$.

Boundary layer thickness is estimated based on the comparison of simulated velocity $v$ and ideal velocity $v_{i d}$ profiles normal to the wall at a certain location.

Table 6.2 Parametric values for RVG

\begin{tabular}{l|l}
\hline Parameter & Value \\
\hline Pitch angle, $\theta\left[^{\circ}\right]$ & 45 \\
\hline Skew angle, $\alpha\left[^{\circ}\right]$ & 30 \\
\hline Height, $\mathrm{h} / \delta[-]$ & 0.36 \\
\hline Diameter, D/ $\delta[-]$ & 0.20 \\
\hline Spacing, W/D [-] & 10 \\
\hline
\end{tabular}


The ideal velocity profile can be obtained from the isentropic relation given in Eq. (6.2) for a constant total pressure $P_{0}$ (the value outside of the boundary layer):

$$
\mathrm{v}_{\mathrm{id}}=\left[\left(\left(\frac{\mathrm{P}_{0}}{\mathrm{P}}\right)^{\frac{\gamma-1}{\gamma}}-1\right) 2 \mathrm{C}_{\mathrm{p}} \mathrm{T}\right]^{1 / 2}
$$

The boundary layer thickness, $\delta$, is defined as the distance normal to the wall at which $v=0.99 \cdot v_{i d}$. In Eq. (6.2), $\mathrm{T}$ is static temperature and $\mathrm{P}$ is static pressure. Coefficient $C_{p}$ is the heat capacity of the gas at constant pressure and $\gamma$ the heat capacity ratio. The boundary layer thickness $\delta$ at the rod location for the reference case $13.9^{\circ}$ amounts to $\delta \approx 0.0216 \cdot c$ and RVGs are scaled according to parameters established in Table 6.2.

Due to the small spacing among rods $(\mathrm{W} \approx 0.043 \cdot c)$ a large amount of them would be needed to cover the entire spanwise length of the experimental models employed by Somers (1997) and Ramsay et al. (1995). Besides, a large grid clustering is needed in the vicinities and downstream of the rod location in order to properly capture the development of streamwise vorticity. Additionally, the complexity of the model to be solved increases rapidly, and the computational resources needed for the full $3 \mathrm{D}$ configuration would be far beyond the available resources. Therefore, the 3D computational domain was limited to a slice of the original experimental model, inside which a single rod was placed and the periodic boundary conditions were applied in the spanwise direction, at lateral surfaces. The RVGs and aerofoil surface were modelled as walls (Fig. 6.14). The proximities of the RVG are resolved by means of a butterfly mesh topology.

The computational domain was divided into a total of 29 blocks. Full non matching boundary (FNMB) connections were applied, so the final number of mesh cells is $3.3 \times 10^{6}$. The NMB connections were applied at an approximate distance of $0.13 \cdot c$ from the aerofoil wall (Fig. 6.14).
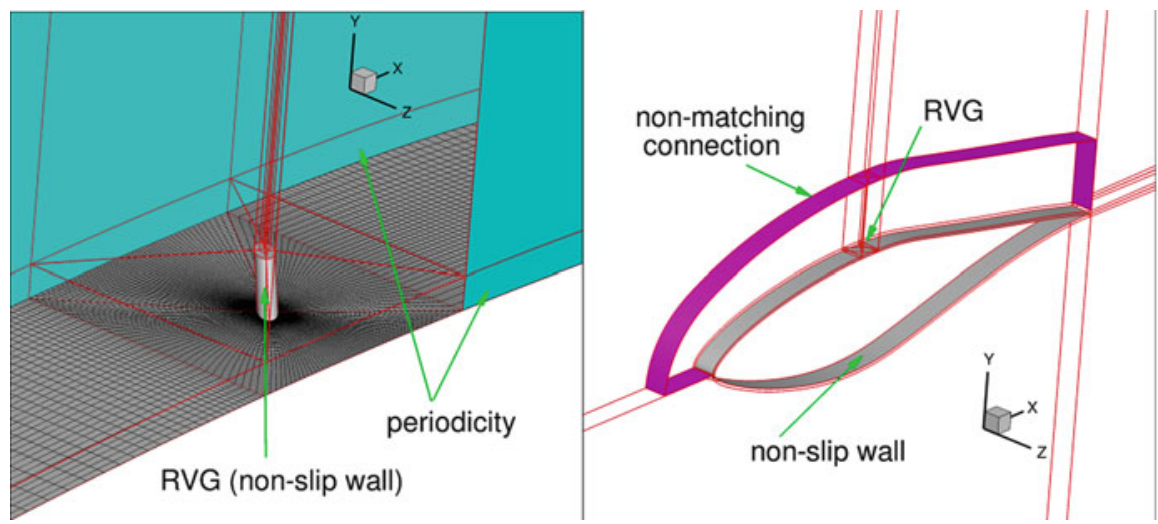

Fig. 6.14 Topology and boundary conditions for flow control case 
In order to avoid the influence of grid nodes distribution in the streamwise direction for the case with and without RVG, the mesh employed for both cases is the same apart from the blocks with the RVG presence. Thus, the S809 aerofoil surface is defined by $737 \times 145 \times 33$ nodes in the wrap-around, normal and spanwise directions, respectively.

Lift $\mathrm{c}_{l}$ and drag $\mathrm{c}_{d}$ coefficients as a function of the angle of attack, obtained numerically for both cases (with and without RVG), are compared in Fig. 6.15. It is shown that for the selected flow cases the application of RVGs provides not only a higher lift force, but also leads to slight reduction of drag. The maximum improvement is observed for an angle of attack of $12.2^{\circ}$, where the lift coefficient $\mathrm{c}_{l}$ is increased by $5.2 \%$ with respect to the clean case and drag is reduced by $1.2 \%$ $\left(\Delta c_{l}=5.2 \%, \Delta c_{d}=-1.2 \%\right)$.

It is important to notice that the RVGs dimensions and spacing are kept constant at the defined location while the increasing inflow angle influences on boundary layer development, so the boundary layer thickness $\delta$ is not the same for all cases. Thus, for an angle of attack of $11.2^{\circ}$ the ratio RVGs height to boundary layer thickness is approximately $h / \delta \approx 0.47(\delta \approx 0.0165 \cdot c)$ and for $12.2^{\circ}, h / \delta \approx 0.42$ $(\delta \approx 0.0185 \cdot c)$, thus verifying the low profile VGs condition $(h / \delta<0.65)$ defined by Rao and Kariya (1988).

\subsubsection{RVGs Chordwise Location}

RVGs were initially located slightly upstream of the separation onset for an angle of attack of $13.9^{\circ}$, at the chordwise position $x_{R V G} / c=0.50$, and designed according to the local boundary layer height using the parametric values summarized in Table 6.2. Notwithstanding the aerodynamic performance improvement obtained by implementing the RVGs at this location, it is prudent to conduct a study of the chordwise location effect on the devices' effectiveness. The flow control devices should be neither positioned too close to the separation (not enough space to develop vortical structures), nor too far away (vorticity diffusion). Four additional
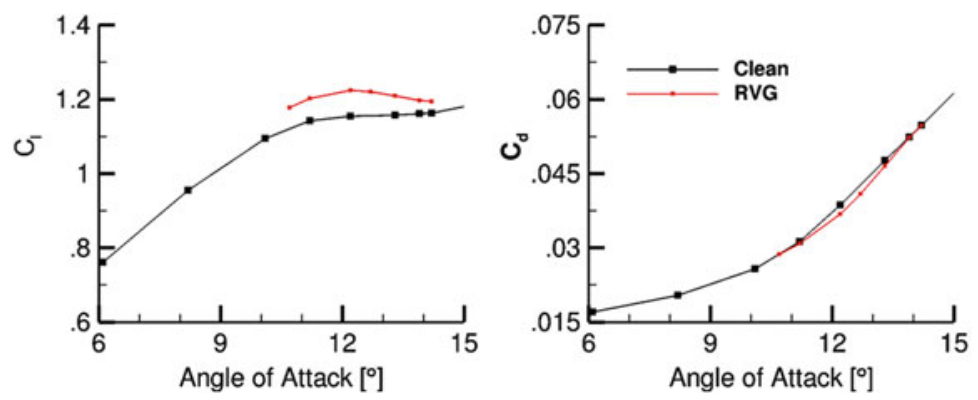

Fig. 6.15 Lift $\boldsymbol{c}_{\boldsymbol{l}}$ and drag $\boldsymbol{c}_{\boldsymbol{d}}$ coefficients vs angle of attack for clean and flow control cases 
chordwise locations $\left(x_{R V G} / c=0.35,0.40,0.45\right.$ and 0.55$)$ were considered and rods dimensioned in relation to the local boundary layer height at each one of the four chordwise locations for a $13.9^{\circ}$ inflow angle. The spacing between rods (W) was kept the same as for the initial configuration. The number of grid nodes and spacing was also preserved-except in the region of the device location, where the refinement is adjusted in order to diminish the grid influence.

A comparison was done for four angles of attack 11.2, 12.2, 13.3 and $13.9^{\circ}$. As mentioned above, increasing the inflow angle from 11.2 to $13.9^{\circ}$ enforces earlier separation, so flow control devices should be located further upstream in order to improve aerofoil performance.

Lift $c_{l}$ and drag $c_{d}$ variations for the considered RVG locations and inflow angles, in respect to the case without flow control, are shown in Fig. 6.16. The values indicate configurations for which the positive or negative effect is obtained. It can be observed that a lift increase is obtained for all locations and inflow angles, except for the angle of attack $13.9^{\circ}$ and $x_{R V G} / c=0.55$, where RVG is located inside the separation bubble. A reduction of drag is obtained for certain rods locations and angles of attack. However, the configuration of maximum lift increase corresponds with the area of drag reduction, leading to aerodynamic improvement.

When the angle of attack is $11.2^{\circ}$, the maximum lift is for RVGs located at $x_{R V G} / c=0.55$. The lift decreases as these are moved closer to the leading edge. Simultaneously, as the RVG location is moved upstream, the drag increases. This means that even if the higher vorticity is created by the RVG located further upstream, the streamwise vortices would dissipate faster than if they were located closer to separation.

A similar effect to the one analysed above is obtained for an inflow angle of $12.2^{\circ}$. The maximum improvement in aerodynamic performance by the implementation of flow control devices is obtained for $x_{R V G} / c=0.50$.

As the angle of attack increases to $13.3^{\circ}$ the separation line moves to $\mathrm{x} / \mathrm{c}=0.55$ and the optimum RVG placement is at the mid-chord. Further upstream locations provide smaller increases in lift (vortices are more dissipated) and higher drag. For
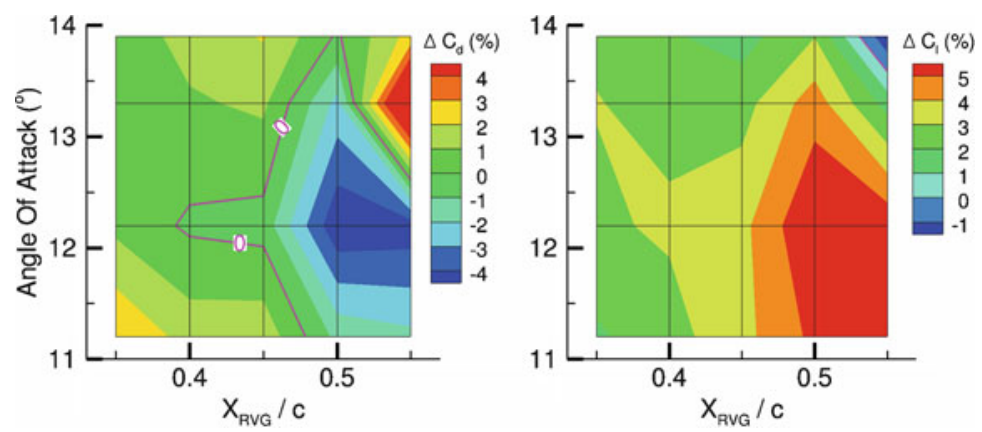

Fig. 6.16 Effect of RVGs chordwise location $x_{R V G} / \boldsymbol{c}$ on $c_{l}$ and $\boldsymbol{c}_{\boldsymbol{d}}$ coefficients 
$x_{R V G} / c=0.55$, the lack of distance to the separation onset impedes the development of the vortex structure and results in a high drag penalty.

Further increasing of the angle of attack up to $13.9^{\circ}$, leads to a more modest profit with the RVG inclusion. The RVGs located at $x_{R V G} / c=0.55$ show a negative effect on the aerodynamic performance-which is expected since the devices are located inside the region of detached flow (separation onset is at $x / c=0.53$ ). At this inflow angle only RVGs located at mid-chord provide a drag reduction.

Based on the contour map analysis, one can conclude that for the selected spanwise distance, $\mathrm{W} \approx 0.043 \cdot c$, the optimum $\mathrm{RVG}$ location is at mid-chord, $x_{R V G} / c=0.50$.

\subsubsection{RVGs Spanwise Separation}

Experimental investigations were conducted to assess the effectiveness of RVGs by varying different parameters; based on the results of these experimental investigations, it was found that the most effective spanwise distance (W) to rod diameter (D) ratio is equal to 10. Consequently, this spanwise distance was used together with the other parameters summarized in Table 6.2 for the initial RVG design. However, due to another flow conditions investigated in the presented chapter, the four RVG spacing scenarios ( $W / D=7.5,10,15$ and 20) are analysed in order to determine a possible influence on separation. The remaining parameters are kept unchanged. Grid influence was minimised by keeping the same number of nodes-except in the spanwise direction, where the number of cells was adjusted according to the increased spanwise distance.

In Fig. 6.17, contour maps of skin friction coefficient $c_{f}$ on the suction side of the S809 aerofoil, at the angle of attack $11.2^{\circ}$, are shown. The case without RVG is shown at the top; below, the cases for rising RVG spanwise distance are compared. The skin friction provides information on the shear stresses distribution and the presence of separation. Additionally, streamlines displayed on top of the skin friction contours allow one to assess the development of flow patterns on the aerofoil. In the presented flow control cases, RVGs were located at the optimum chordwise position previously identified (Sect. 6.5.2): $x_{R V G} / c=0.50$.

It is clearly visible in Fig. 6.17 that skin friction along the chord increases downstream of the RVGs' location and it is higher than in the case of "uncontrolled" flow. Narrow regions of lower friction are present in the zones between rods. This structure is originated by the streamwise vortices. The convected vortices transport momentum from the outer region of the boundary layer to the layer closer to the wall on one vortex side, while lifting the boundary layer on the opposite one.

Assuming a spanwise distance equal to 10 rod diameters (reference configuration), the region of detached flow existing in the clean case vanishes and shrunken backflow zones appear between RVGs at around $75 \%$ of the chord. It is also clearly visible that a spanwise velocity component is generated by the RVGs, rooted in the spanwise migration of the created vortices. 

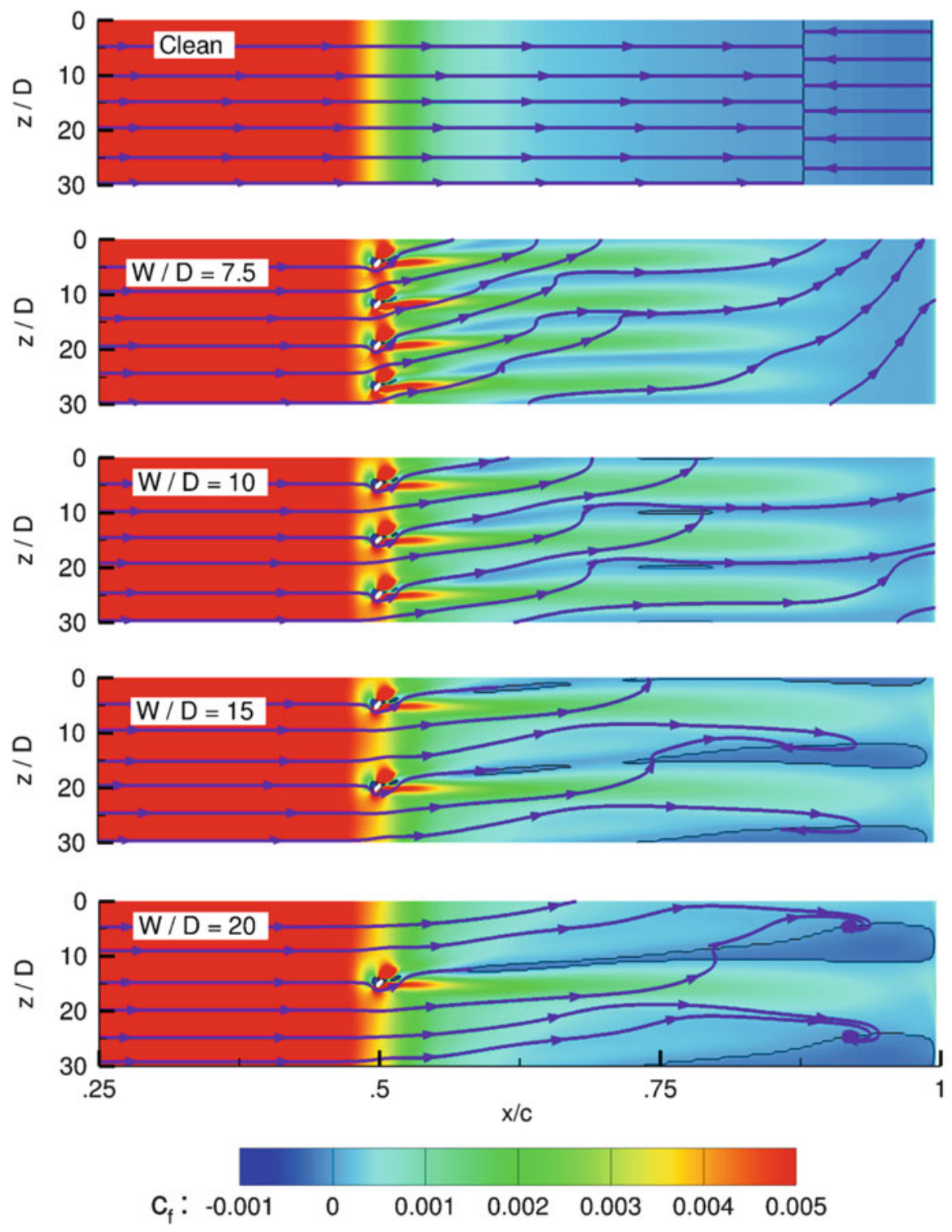

Fig. 6.17 Skin friction coefficient $c_{f}$ and surface streamlines for different RVGs spanwise distance $W / D$

When the distance is decreased to 7.5 diameters, the small separation bubbles between streamwise vortices vanished and no detached flow is noticed. Notwithstanding this fact, the skin friction contour map reveals that the penetration of the streamwise vorticity has been shortened in comparison with the reference configuration $(10 \cdot D)$. Consequently, a possible interaction of streamwise vortices 

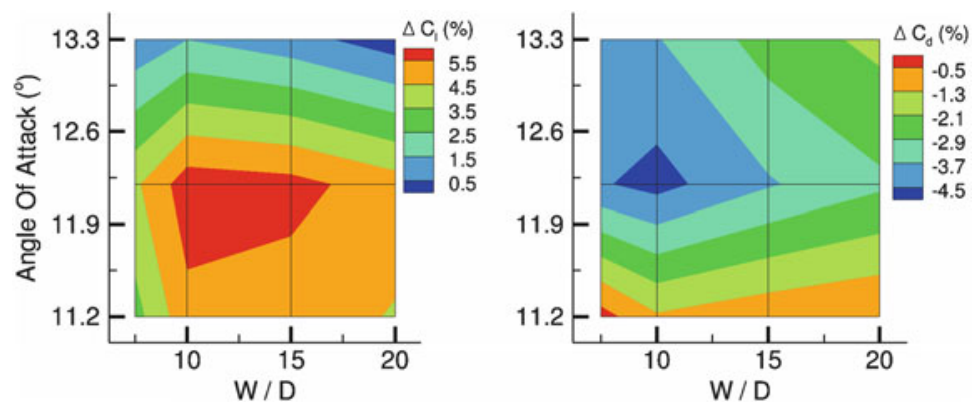

Fig. 6.18 Effect of RVGs spanwise separation $W / D$ in $\mathbf{c}_{\boldsymbol{l}}$ and $\mathbf{c}_{\mathbf{d}}$ coefficients

appeared in the flow for a distance $7.5 \cdot D$ which could decrease the RVG effectiveness.

As the separation between rods increases from the reference to 15 diameters, the length and width of the backflow bubbles located between rods also increases. The separation onset is moved upstream to $70 \%$ of the chord. Furthermore, for this RVG configuration, the trailing edge separation was only partially eliminated and the elongated regions of detached flow in the proximities of the trailing edge still exist. For a further rods spacing increase to $20 \cdot D$, one can observe that the previously described backflow bubbles have merged into a long recirculation region. In any case, the global separated area is still lower compared to a case without RVG.

In Fig. 6.18, the contourmaps of lift $c_{l}$ and drag $c_{d}$ variations with respect to the clean case for RVGs at chordwise location $x_{R V G} / c=0.5$ are presented. It is clearly shown that for an angle of attack of $11.2^{\circ}$ a separation of 7.5 rod diameters provides the minimum drag reduction and lift increase, which strengthen the previous hypothesis of interaction between streamwise vortices. Spanwise distances of $10 \cdot D$ and $15 \cdot D$ provide the highest increases of lift coefficient for all angles of attack. However, the implementation of a spanwise distance $10 \cdot D$ allows for further decrease of the drag in the studied angles of attack. At $13.3^{\circ}$ the separation has moves upstream to $\mathrm{x} / \mathrm{c}=0.55$ and the decay of maximum streamwise vorticity in chordwise direction is more intensive than for lower angles of attack. Nevertheless, aerodynamic improvement obtained by means of implemented RVG is still positive, although the global extremum is found for $W=10 \cdot D$.

\subsection{Conclusions}

The numerical simulations carried out for NREL Phase VI rotor configurations indicated a good agreement of obtained results with experimental data. This validated the model, and proved its capability to predict aerodynamic performance. Hence, the validated model was used to investigate rod vortex generators as a means of improving aerodynamic performance. The effect of RVGs and generated 
streamwise vortices on the separation reduction was analysed on a S809 aerofoil. Numerical simulations for a blade section defined by the S809 aerofoil were also validated by experimental data, and the results were considered as the reference case for the flow control analysis. A study was conducted to analyse the effect of varying the location and spanwise direction of rod vortex generators: the subsequent numerical results confirmed the effectiveness of RVGs as a means of flow separation reduction and increasing aerodynamic performance.

Acknowledgments The authors would like to acknowledge the European Commission for their research grant under the project FP7-PEOPLE-2012-ITN 309395 "MARE-WINT" (new MAterials and REliability in offshore WINDd Turbines technology). The research was supported in part by the PL-Grid Infrastructure and TASK Supercomputing Centre in Gdansk.

Open Access This chapter is distributed under the terms of the Creative Commons AttributionNonCommercial 4.0 International License (http://creativecommons.org/licenses/by-nc/4.0/), which permits any noncommercial use, duplication, adaptation, distribution and reproduction in any medium or format, as long as you give appropriate credit to the original author(s) and the source, provide a link to the Creative Commons license and indicate if changes were made.

The images or other third party material in this chapter are included in the work's Creative Commons license, unless indicated otherwise in the credit line; if such material is not included in the work's Creative Commons license and the respective action is not permitted by statutory regulation, users will need to obtain permission from the license holder to duplicate, adapt or reproduce the material.

\section{References}

Amitay M, Smith BL, Glezer A (1998) Aerodynamic flow control using synthetic jet technology. In: Abstracts of the 36th AIAA aerospace sciences meeting exhibit, Reno, 12-15 January 1998

Andersen PB, Henriksen L, Gaunaa M et al (2010) Deformable trailing edge flaps for modern megawatt wind turbine controllers using strain gauge sensors. Wind Energy 13:193-206

Bak C, Bitsche R, Yde A et al (2012) Light rotor: the 10-MW reference wind turbine. Paper presented at the EWEA 2012 - European wind energy conference and exhibition, Copenhagen, 16-19 April 2012

Choi YH, Merkle CL (1993) The application of preconditioning in viscous flows. J Comput Phys 105(2):207-223

Doerffer P, Flaszyski P, Szwaba R (2009) Rod patent. Poland patent PL38968

Eppler R, Somers DM (1980) A computer program for the design and analysis of low-speed airfoils. In: NASA Technical Reports. Available via NASA Technical Reports Server (NTRS) http://hdl.handle.net/2060/19810005410. Accessed 07 Apr 2016

Gao L, Zhang H, Liu Y et al (2015) Effects of vortex generators on a blunt trailing-edge airfoil for wind turbines. Renew Eneryg 76:303-311

Hakimi N (1997) Preconditioning methods for time dependent Navier-Stokes equations: application to environmental and low speed flows. Dissertation, Vrije Universiteit

Hand MM, Simms DA, Fingersh L et al (2001) Unsteady aerodynamics experiment phase VI: wind tunnel test configurations and available data campaigns: NREL/TP-500-29955. In: National Renewable Energy Laboratory (NREL) Publications. Available via NREL. http://www.nrel. gov/docs/fy02osti/29955.pdf. Accessed 06 Apr 2016 
Jameson A (1991) Time dependent calculations using multigrid, with applications to unsteady flows past airfoils and wings. In: Abstracts of the 10th computational fluid dynamics conference, fluid dynamics and co-located conferences, AIAA, Honolulu, 24-26 June 1991

Johnson SJ, Baker JP, Van Dam CP et al (2010) An overview of active load control techniques for wind turbines with an emphasis on microtabs. Wind Energy 13:239-253

Kostas J, Foucaut JM, Stanislas M (2007) The flow structure produced by pulsed-jet vortex generators in a turbulent boundary layer in an adverse pressure gradient. Flow Turbul Combust 78:331-363

Lin JC (2002) Review of research on low-profile vortex generators to control boundary-layer separation. Prog Aerosp Sci 38:389-420

Lindenburg C (2003) Investigation into rotor blade aerodynamics: analysis of the stationary measurements on the UAE phase-VI rotor in the NASA-AMES wind tunnel ECN-C-03-025. In: Energy Research Centre of the Netherlands (ECN) Publications. Available via ECN. https:// www.ecn.nl/publications/PdfFetch.aspx?nr=ECN-C--03-025. Accessed 07 Apr 2016

Martinez J, Doerffer P, Szulc O et al (2015a) Aerodynamic analysis of wind turbine rotor blades. Task Q 19(2):129-140

Martinez J, Doerffer P, Szulc O (2015b) CFD validated technique for prediction of aerodynamic characteristics on horizontal axis wind energy turbines. Poster presented at the Offshore 2015 Conference, The European Wind Energy Association, Copenhagen, 10-12 March 2015

McManus K, Legner HH, Davis SJ (1994) Pulsed vortex generator jets for active control of flow separation. In: Abstracts of the fluid dynamics conference, AIAA, Colorado Springs, 20-23 June 1994

Menter FR (1994) Two-equation eddy-viscosity turbulence models for engineering applications. AIAA J 32(8):1598-1605

Menter FR, Garbaruk AV, Egorov Y (2012) Explicit algebraic Reynolds stress models for anisotropic wall-bounded flows. Prog Flight Phys 3:89-104

Nelson RC, Corke TC, Othman H (2008) A smart wind turbine blade using distributed plasma actuators for improved performance. In: Abstracts of the 46th AIAA aerospace sciences meeting and exhibit, Reno, 7-10 January 2008

Paul AR, Joshi S, Jindal A et al (2013). Comparison active-passive: experimental studies of active and passive flow control techniques applied in a twin air-intake. Sci World J. doi:10.1155/2013/523759

Ramsay RR, Hoffman MJ, Gregorek GM (1995) Effects of grit roughness and pitch oscillations on the S809 airfoil NREL/TP-442-7817. In: National Renewable Energy Laboratory Reports. Available via NREL. https://wind.nrel.gov/airfoils/OSU_data/reports/3x5/s809.pdf. Accessed 07 Apr 2016

Rao DM, Kariya TT (1988) Boundary-layer submerged vortex generators for separation controlan exploratory study. In: Abstracts of the 1st national fluid dynamics conference, AIAA, Cincinnati, 25-28 July 1988

Somers DM (1997) Design and experimental results for the S809 airfoil NREL/SR-440-6918. NREL Report: In: National Renewable Energy Laboratory Reports. Available via NREL. www.nrel.gov/docs/legosti/old/6918.pd. Accessed 07 Apr 2016

Sørensen NN, Michelsen JA (2000) NREL/NWTC aerodynamics code blind comparison. In: Abstracts of the 2 science panel meeting, NREL Boulder, Colorado, 5-6 December 2000

Spalart PR, Allmaras SR (1992) A one-equation turbulence model for aerodynamic flows. In: Abstracts of the 30th AIAA aerospace sciences meeting and exhibit, AIAA, Reno, 6-9 January 1992

Sutherland W (1893) LII. The viscosity of gases and molecular force. Philos Mag Ser 5 36(223):507-531

Taylor HD (1947) The elimination of diffuser separation by vortex generators. United Aircraft Corporation Research Department Report No. R-4012-3

Tejero F, Doerffer P, Szulc O (2015) Shock wave induced flow separation control by air-jet and rod vortex generators. Task Q 19(2):167-180 
van Dam CP, Chow R, Zayas JR et al (2007) Computational investigations of small deploying tabs and flaps for aerodynamic load control. J Phys Conf Ser. doi:10.1088/1742-6596/75/1/012027 van Dam CP, Berg DE, Johnson SJ (2008) Active load control techniques for wind turbines. In: Department of Energy Scientific and Technical Information (OSTI). Available via OSTI. http:// www.osti.gov/scitech/servlets/purl/943932. Accessed 07 Apr 2016

Wagner S, Bareiss R, Guidati G (eds) (2012) Wind turbine noise. Springer, Berlin

Wallis RA, Stuart CM (1962) On the control of shock induced boundary layer separation with discrete air jets. In: National Advisory Committee for Aeronautics (NACA) Archives. Available via NACA. http://naca.central.cranfield.ac.uk/reports/arc/cp/0595.pdf. Accessed 07 Apr 2016

Wiltse JM, Glezer A (1993) Manipulation of free shear flows using piezoelectric actuators. J Fluid Mech 249:261-285 